УДК 621.372.8.082.5

\author{
А.В. Бурдин, В.А. Бурдин, В.А. Андреев \\ Поволжский государственный университет телекоммуникаций \\ и информатики, Самара, Россия \\ МОДЕЛИРОВАНИЕ КВАРЦЕВЫХ МНОГОМОДОВЫХ
ОПТИЧЕСКИХ ВОЛОКОН ТРАНСПОРТНЫХ СЕТЕЙ \\ СВЯЗИ НОВОГО ПОКОЛЕНИЯ
}

\begin{abstract}
На сегодняшний день нелинейный предел Шеннона фрактически определил порог применения одномодовых оптических волокон (OB) на протяженных волоконно-оптических линиях передачи высокоскоростных транспортных сетей связи нового поколения. Одним из кардинальных способов подавления нелинейных эффектов в оптическом линейном тракте является непосредственное уменьшение нелинейности самого волоконного световода за счет существенного увеличения диаметра его сердцевины. В работе представлен альтернативный поход к моделированию профиля показателя преломления таких кварцевых ОВ с увеличенным до 50 мкм диаметром сердцевины, что соответствует типовым многомодовым ОВ, с уменьшенной дифференциальной модовой задержкой, оптимизированным при этом для передачи сигналов в телекоммуникационных «C» и «L» волновых диапазонах.

Ключевые слова: маломодовые и многомодовые оптические волокна, оптические волокна с увеличенным диаметром сердцевины, дифференциальная модовая задержка, нелинейный предел Шеннона.
\end{abstract}

\author{
A.V. Burdin, V.A. Burdin, V.A. Andreev \\ Povolzhskiy State University of Telecommunications and Informatics, Samara, \\ Russian Federation

\section{DESIGN OF SILICA MULTIMODE FIBERS FOR NEXT GENERATION TRANSPORT NETWORKS}

Nowadays nonlinear Shannon limit defines the bounds of conventional single mode optical fiber application for long-haul fiber optic links of the next generation high bit rate transport networks. One of the approaches for suppression of nonlinear effects in optical trunk is directly reducing of fiber nonlinearity by the great expanding of core diameter. We present alternative method for design of refractive index profile for silica $\mathrm{GeO}_{2}$-doped optical fibers with expanded core up to $50 \mu \mathrm{m}$ that corresponds to conventional multimode optical fibers with low differential mode delay for operating over "C" and "L"- bands.

Keywords: few-mode and multimode optical fibers, optical fibers with expanded core, differential mode delay, nonlinear Shannon limit. 


\section{1. Вопросы выбора конструкции оптических волокон транспортных сетей связи нового поколения}

На сегодняшний день нелинейный предел Шеннона фактически определил порог пропускной способности и, соответственно, ограниченные возможности применения одномодовых оптических волокон (ОВ) на протяженных волоконно-оптических линиях передачи (ВОЛП) высокоскоростных транспортных сетей связи нового поколения, которые, согласно прогнозам аналитиков, к 2020 г. должны поддерживать скорости передачи десятки терабит в секунду и более [1-7].

Очевидным кардинальным способом подавления нелинейных эффектов в оптическом линейном тракте является непосредственно уменьшение нелинейности самого волоконного световода. В этом смысле радикальным решением данной проблемы является переход на волокна новой конструкции, например: $\mathrm{OB}$, с полой сердцевиной, микроструктурированные/дырчатые/фотонно-кристаллические ОВ в разных вариантах конструктивного исполнения, а также многосердцевинные ОВ, с которыми связывают большие перспективы применения на высокоскоростных сетях в сочетании с технологией пространственного мультиплексирования (SDM - Space Division Multiplexing) [2, 3, 7-10]. Однако широкое внедрение таких волоконных световодов на сетях связи в ближайшем будущем пока остается под вопросом. Во-первых, это связано с проблемой стабильности геометрии сложной конструкции $\mathrm{OB}$, особенно с точки зрения магистральных ВОЛП, протяженность которых исчисляется сотнями километров. Во-вторых, по-прежнему сохраняется высокая стоимость таких световодов: по сравнению с кварцевыми ОВ традиционной конструкции разница может достигать 2 порядка и даже более. В-третьих, совместное сочетание сложных/мультикомпонентных, в частности многосердцевинных, ОВ в сочетании с SDM потребует увеличения числа компонентов системы по числу «пространственных» каналов - сердцевин, например оптических усилителей или перестраиваемых волновых мультиплексоров ввода/вывода.

Для волокон традиционной конструкции уменьшение нелинейности в общем случае может достигаться либо путем выбора соответствующего материала сердцевины, либо за счет увеличения площади эффективного сечения, в том числе благодаря увеличению диаметра сердцевины. Однако с точки зрения одномодовых ОВ последнее ограничено условиями обеспечения одномодового режима. По этой 
причине даже специализированные одномодовые ОВ с увеличенной площадью эффективного сечения позволяют снизить нелинейность не более чем на 5-6 \% по сравнению со стандартными одномодовыми OB $[6,11]$.

Большие перспективы связывают с технологией SDM на модовых компонентах в сочетании с так называемыми маломодовыми OB, поддерживающими распространение не более 3-6 направляемых мод [2, 3, 7]. Однако и в этом случае, с учетом того, что площадь эффективного сечения типового маломодового ОВ составляет около 130-160 мкм² в зависимости от порядка рассматриваемой моды, а стандартного одномодового ОВ - 85 мкм ${ }^{2}$, при прочих равных условиях интенсивность оптического излучения в таком маломодовом ОВ снижается по сравнению с одномодовым всего лишь примерно в 1,5 раза $[2,3,7,12,13]$.

Радикальное увеличение диаметра сердцевины, позволяющее практически нивелировать нелинейность ОВ, неизбежно приводит к появлению еще большего числа новых модовых составляющих высших порядков. Не случайно в некоторых публикациях, посвященных технологии SDM, например работе [7], декларируется невозможность использования ОВ с сильно увеличенным радиусом сердцевины, поскольку в этом случае последние поддерживают распространение большого числа направляемых мод и фактически становятся многомодовыми.

Вместе с тем следует отметить, что при «классическом» многомодовом режиме оптический сигнал содержит до 1000 и более модовых составляющих $[14,15]$. В то время как передача оптического сигнала, возбуждаемого когерентным источником излучения - лазером, по ОВ даже с намного увеличенным, относительно размеров одномодовых ОВ, диаметром сердцевины осуществляется в маломодовом режиме, когда число модовых компонент ограничено несколькими десятками $[14,15]$.

Например, согласно проведенным ранее теоретическим и экспериментальным исследованиям [17], для типового кварцевого градиентного многомодового ОВ категории ОМ2+/OM3, диаметр сердцевины которого составляет 50 мкм, в переносе оптического сигнала, генерируемого лазерным источником в области длины волны 1310 нм при центрированном вводе, даже с учетом процессов взаимодействия и смешения мод, возникающих за счет нерегулярной структуры и изгибов ОВ, будет участвовать не более 16 модовых составляющих, что 
фактически делает его маломодовым. При этом площадь эффективного

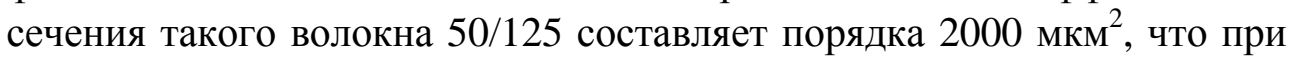
прочих равных условиях обеспечивает снижение интенсивности оптического излучения в нем, по сравнению с одномодовыми ОВ, уже более чем в 20 раз. В результате для подобных волоконных световодов на передний план выходят линейные искажения. Последние в несколько раз превышают линейные искажения в одномодовом ОВ. Однако при соответствующих условиях, в частности сохранении огибающей оптического импульса, эти негативные эффекты могут быть устранены традиционными средствами адаптивной компенсации линейных искажений, в том числе с помощью таких известных технологий, как упреждающая коррекция ошибок и электронная компенсация дисперсии. Все это делает перевод волоконно-оптических магистралей на маломодовые ВОЛП на основе многомодовых ОВ 50/125 традиционной конструкции, функционирующих в маломодовом режиме, в сочетании с технологиями SDM на модовых компонентах и MIMO (Multiple Input- Multiple Output - система с множественными входами/выходами) одним из перспективных направлений реализации высокоскоростных транспортных сетей связи нового поколения.

В этом смысле ключевым фактором, сдерживающим эффективное применение многомодовых ОВ в рамках данного приложения, является дифференциальная модовая задержка (DMD - Differential Mode Delay), которая сильно искажает форму импульса на приемной стороне, проявляясь в виде разделения сигнала на отдельные компоненты. В данной работе представлен альтернативный поход к моделированию профиля показателя преломления таких кварцевых ОВ с увеличенным до 50 мкм диаметром сердцевины, что соответствует типовым многомодовым OB, с уменьшенной DMD и оптимизированным при этом для передачи сигналов в телекоммуникационных «C» и «L» волновых диапазонах.

\section{2. Обзор методов синтеза профилей показателя преломления многомодовых ОВ с увеличенной полосой пропускания}

Достаточно подробный обзор известных решений по разработке многомодовых ОВ с увеличенной полосой пропускания, ориентированных не только на уменьшение модовой дисперсии, но и на снижение DMD в маломодовом режиме, представлен в монографии [18]. Большинство подходов в основном базируются на мониторинге DMD 
еще на стадии формирования заготовки волокна. Наибольший интерес представляют работы [19-24].

Так, в [19-21] целевая функция оценивает степень близости искомого профиля к некоторому профилю, наилучшему с точки зрения минимального разброса групповых скоростей всей совокупности мод, которые могут быть переданы по многомодовому OB, т.е. для многомодового режима передачи.

Вместе с тем в работах [22-24] в качестве целевой функции принята разность между интегральным или локальным значением параметра $\alpha_{i}$ аппроксимации профиля заготовки степенной функцией и значением параметра степенного профиля $\alpha$, оптимального с точки зрения многомодового режима передачи. При этом сама локальная коррекция параметра градиента $\alpha$ выполняется на основе воспроизведения «оптимальной» диаграммы DMD. Последняя представляет собой распределение значений DMD по соответствующим порядкам направляемых мод, пересчитанным на обобщенное модовое число, и формируется путем соответствующей модификации исходной диаграммы DMD базового модельного волокна, построенной в условиях равномерного возбуждения. В то же время известно [25], что именно центрированный ввод является одним из эффективных и доступных для реализации в полевых условиях способов увеличения полосы пропускания многомодовых ВОЛП, функционирующих в маломодовом режиме передачи сигнала. Однако нельзя не отметить, что данный подход позиционировался как решение для реализации так называемых универсальных многомодовых ОВ, оптимизированных для работы с лазерными источниками, во всех трех окнах прозрачности: 850, 1310 и 1550 нм соответственно.

Вышесказанное позволяет сформулировать основные факторы, которые в обязательном порядке должны быть учтены при синтезе профиля многомодового ОВ с пониженным значением DMD - так называемых волокон LDMDF (Low DMD Fibers), оптимизированных для функционирования в маломодовом режиме:

1) в качестве начального приближения целесообразно использовать конструкцию, геометрию, профиль показателя преломления и его параметры реальных апробированных образцов многомодовых ОВ категории ОМ2+...ОM4, но никак не волокон первых поколений категорий ОМ1/OM2, а также модельных ОВ с идеализированными степенными профилями показателя преломления; 
2) разработка или модификация конструкции/профиля показателя преломления ОВ должна в обязательном порядке базироваться на сопоставлении с диаграммой или профилем DMD;

3) в обязательном порядке необходимо учитывать тип лазера, состав поперечных мод его излучения и условия ввода сигнала в линию.

Наконец, в контексте данной работы нельзя не отметить, что уменьшенное значение DMD должно обеспечиваться не на одной оптической несущей, а в телекоммуникационных волновых диапазонах $\ll \mathrm{C} »$ и $\ll \mathrm{L} »$.

\section{3. Моделирование волокон LDMDF}

Предлагаемое решение базируется на выборе специализированной формы профиля показателя преломления, обеспечивающей выравнивание задержек направляемых мод заданного порядка относительно некоторого опорного значения $t_{\mathrm{BASE}}$. Конструкция предлагаемого ОВ, которое в общем случае удовлетворяет условиям приближения слабонаправляющего оптического волновода, является традиционной: представляет собой кварцевую сердцевину, легированную германием $\left(\mathrm{SiO}_{2}-\mathrm{GeO}_{2}\right)$, диаметр которой составляет 50 мкм, окруженную одной внешней сплошной оболочкой из чистого кварца $\mathrm{SiO}_{2}$. В отличие от известных решений [18-24], для описания искомого профиля используется общий подход метода стратификации [26]. В результате моделируемый слабонаправляющий волоконный световод с произвольным осесимметричным профилем показателя преломления, ограниченным одной внешней сплошной оболочкой, рассматривается как слабонаправляющий волоконный световод с многослойным профилем показателя преломления. При этом искомый профиль в области сердцевины ОВ представляется в виде конечного числа $N$ слоев, в пределах которых значение показателя преломления остается постоянным:

$$
n(R)= \begin{cases}n_{k}, & R_{k}=\frac{k}{N}, \quad 0 \leq k \leq N-1, \\ n_{N}, & 1<R \leq+\infty\end{cases}
$$

а сама функция $f(R)$, описывающая профиль показателя преломления:

$$
n^{2}(R)=n_{\max }^{2}[1-2 \Delta \cdot f(R)],
$$


записывается в следующем виде:

$$
f(R)= \begin{cases}h_{k}, & R_{k}=\frac{k}{N}, \quad 0 \leq k \leq N-1, \\ 1, & 1<R \leq+\infty,\end{cases}
$$

где $h_{k}$ - локальный параметр профиля, $h_{k}=\left(n_{\max }^{2}-n_{k}^{2}\right) /\left(n_{\max }^{2}-n_{N}^{2}\right) ; n_{k}-$ показатель преломления $k$-го промежуточного слоя $(k=0,1,2 \ldots N-1)$; $n_{\max }$ - максимум показателя преломления в области сердцевины; $n_{N}$ - показатель преломления внешней сплошной оболочки; $\Delta=\left(n_{\max }^{2}-n_{N}^{2}\right) / 2 n_{\max }^{2}-$ параметр высоты профиля; $R_{k}-$ нормированная радиальная координата $k$-го промежуточного слоя, $R_{k}=r_{k} / a$; $r_{k}$ - радиальная координата $k$-го промежуточного слоя; $a$ - радиус сердцевины моделируемого ОВ.

В результате искомая форма профиля показателя преломления в пределах области сердцевины волокна LDMDF выбирается таким образом, чтобы обеспечивалась минимизация некоторой величины $F$, которая описывается выражением вида

$$
F=\sum_{j=1}^{M}\left(t_{d(j)}-t_{\mathrm{BASE}}\right)^{2},
$$

где $t_{d(j)}$ - искомое значение задержки $j$-й направляемой моды азимутального $l$ и радиального $m$ порядка $L P_{l m}{ }^{(j)}$ диаграммы DMD, соответствующей синтезируемому профилю показателя преломления волокна LDMDF; $t_{\text {BASE }}$ - некоторое опорное значение модовой задержки, относительно которого осуществляется выравнивание задержек направляемых мод $L P_{l m}$ заданных порядков; $M$ - число направляемых мод, переносящих мощность маломодового оптического сигнала в многомодовом ОВ, для которых оптический фактор ограничения составляет не менее $0,5\left(P_{c o(j)} \geq 0,5\right)$, а нормированная амплитуда - не менее 0,1 . Значение $M$, главным образом, определяется следующими факторами: условиями ввода сигнала с выхода лазера в многомодовое $\mathrm{OB}$, составом поперечных мод излучения, которое формируется непосредственно на выходе источника и зависит от типа лазера и его соответствующих характеристик, а также прогнозом появления новых модовых компонент, обусловленного нерегулярной структурой ОВ и наличием 
микро- и макроизгибов волокон в кабеле, неизбежно возникающих при инсталляции ВОЛП. При этом, в отличие от известных решений, опорное значение модовой задержки $t_{\mathrm{BASE}}$ предлагается выбирать из диапазона значений диаграммы DMD, построенной, как было отмечено выше, для $M$ направляемых мод заданного порядка, многомодовых ОВ последних поколений ОМ2+...ОМ4.

В процессе минимизации указанной величины $F$ необходимо многократно решать прямую задачу анализа многомодового волоконного световода с направленно изменяемыми параметрами. При этом аргументы целевой функции $F$ представляют собой массив параметров $h_{k}$, полностью описывающий профиль показателя преломления ОВ.

Для этой цели предлагается использовать разработанную ранее модификацию приближения Гаусса [27], обобщенную на случай расчета параметров передачи направляемых мод произвольного порядка, распространяющихся в слабонаправляющих ОВ с произвольным осесимметричным профилем показателя преломления, ограниченным одной внешней сплошной оболочкой, для представления которого также используется описанный выше подход на основе метода стратификации (далее ОМПГ - обобщение модификации приближения Гаусса). Это позволяет перейти от известной интегральной [28] к аналитической форме записи вариационного выражения для квадрата волноводного параметра моды в сердцевине $U^{2}$ в виде конечных вложенных сумм вида [27]:

$$
\begin{gathered}
U^{2}=\frac{(m-1) !}{(l+m-1) !}\left\{\frac{Q}{R_{0}^{2}}+V^{2}\left[X_{0}+\sum_{k=0}^{N-1} h_{k}\left(X_{1}-X_{2}\right)\right]\right\} \\
X_{0}=\exp \left(-\frac{1}{R_{0}^{2}}\right) \sum_{q=0}^{2 m-2} D_{q} \sum_{p=0}^{l+q} \frac{(l+q) !}{p ! R_{0}^{2 p}} \\
X_{1}=\exp \left(-\frac{k^{2}}{N^{2} R_{0}^{2}}\right) \sum_{q=0}^{2 m-2} D_{q} \sum_{p=0}^{l+q} \frac{(l+q) !}{p ! R_{0}^{2 p}} \frac{k^{2 p}}{N^{2 p}} \\
X_{2}=\exp \left(-\frac{(k+1)^{2}}{N^{2} R_{0}^{2}}\right) \sum_{q=0}^{2 m-2} D_{q} \sum_{p=0}^{l+q} \frac{(l+q) !}{p ! R_{0}^{2 p}} \frac{(k+1)^{2 p}}{N^{2 p}}
\end{gathered}
$$


где $R_{0}$ - эквивалентный нормированный радиус пятна моды, $R_{0}=\rho_{0} / a$; $\rho_{0}-$ эквивалентный радиус пятна моды;

$$
\begin{gathered}
Q=\frac{(l+m-1) !(3 l+2 m-1)}{(m-1) !}+2 l^{2} \sum_{q=0}^{2 m-2} D_{q}(q+l-1) !-4 l \sum_{q=0}^{2 m-2} C_{q}(q+l) ! ; \\
D_{q}=\sum_{p=\max (0, q-m+1)}^{\min (q, m-1)} b_{p}^{(l, m-1)} b_{q-p}^{(l, m-1)} ; \\
C_{q}=\sum_{p=\max (0, q-m+1)}^{\min (q, m-1)} b_{p}^{(l, m-1)} b_{q-p}^{(l+1, m-1)},
\end{gathered}
$$

$b_{p}^{(l, m)}$ - соответствующие коэффициенты степенного ряда формулы явного выражения полинома Лагерра $L_{m}^{(l)}(x)$ [29]:

$$
\begin{gathered}
L_{m}^{(l)}(x)=\sum_{q=0}^{m} b_{q}^{(l, m)} x^{q} ; \\
b_{q}^{(l, m)}=(-1)^{q} \frac{(l+m) !}{(l+q) !(m-q) ! q !},
\end{gathered}
$$

где $V$ - нормированная частота (волноводный параметр), определяемая первичными параметрами ОВ, $V=k_{0} a n_{\max } \sqrt{2 \Delta} ; k_{0}$ - волновое число для оптической волны в вакууме, $k_{0}=2 \pi / \lambda ; \lambda$ - рабочая длина волны; $c$ - скорость света в вакууме.

Характеристическое уравнение для эквивалентного нормированного радиуса пятна моды $\partial U^{2} / \partial R_{0}=0$, получаемое согласно общему алгоритму приближения Гаусса [28] в результате дифференцирования (5) по $R_{0}$, также приводится к аналитическому виду:

$$
\begin{gathered}
-Q+V^{2}\left[S_{0}+\sum_{k=0}^{N-1} h_{k}\left(S_{1}-S_{2}\right)\right]=0 ; \\
S_{0}=\exp \left(-\frac{1}{R_{0}^{2}} \sum_{q=0}^{2 m-2} D_{q} \sum_{p=0}^{l+q} \frac{(l+q) !}{p ! R_{0}^{2 p}}\left(1-p R_{0}^{2}\right) ;\right.
\end{gathered}
$$




$$
\begin{gathered}
S_{1}=\exp \left(-\frac{k^{2}}{N^{2} R_{0}^{2}}\right) \sum_{q=0}^{2 m-2} D_{q} \sum_{p=0}^{l+q} \frac{(l+q) !}{p ! R_{0}^{2 p}} \frac{k^{2 p}}{N^{2 p}}\left(\frac{k^{2}}{N^{2}}-p R_{0}^{2}\right) ; \\
S_{2}=\exp \left(-\frac{(k+1)^{2}}{N^{2} R_{0}^{2}}\right) \sum_{q=0}^{2 m-2} D_{q} \sum_{p=0}^{l+q} \frac{(l+q) !}{p ! R_{0}^{2 p}} \frac{(k+1)^{2 p}}{N^{2 p}}\left(\frac{(k+1)^{2}}{N^{2}}-p R_{0}^{2}\right) .
\end{gathered}
$$

Результатом численного решения уравнения (6), записанного для геометрических параметров исследуемого ОВ и дискретных значений азимутального и радиального порядков заданной моды $L P_{l m}$, является эквивалентный нормированный радиус пятна искомой моды $R_{0}$. Последующая подстановка $R_{0}$ в вариационное выражение (5) позволяет выполнить оценку параметра моды в сердцевине $U$, который непосредственно связан с постоянной распространения известным выражением [28]:

$$
\beta^{2}=k_{0}^{2} n_{0}^{2}-\frac{U^{2}}{a^{2}} .
$$

Полученное решение характеристического уравнения (6) с учетом последующей подстановки в (5) и далее в (7) должно удовлетворять условию отсечки направляемых мод $k n_{N}<\beta \leq k n_{0}$ и, как было отмечено выше, неравенству для оптического фактора ограничения $P_{c o}^{(l m)} \geq 0,5$, для которого в рамках приближения слабонаправляющего оптического волновода, с учетом приближения Гаусса, также было получено аналитическое выражение вида

$$
P_{c o}^{(l m)}=\frac{(m-1) !}{(l+m-1) !} \sum_{q=0}^{2 m-2} D_{q}(l+q) !\left[1-\exp \left(-\frac{1}{R_{0}^{2}}\right) \sum_{p=0}^{l+q} \frac{1}{p ! R_{0}^{2 p}}\right] .
$$

Все это позволяет также получить аналитические формулы для производных параметра моды в сердцевине $\partial U^{2} / \partial \lambda$ :

$$
\begin{gathered}
\frac{\partial U^{2}}{\partial \lambda}=\frac{(m-1) !}{(l+m-1) !}\left\{-\frac{2 Q}{R_{0}^{3}} \frac{\partial R_{0}}{\partial \lambda}+\frac{\partial V^{2}}{\partial \lambda}\left[X_{0}+\sum_{k=0}^{N-1} h_{k}\left(X_{1}-X_{2}\right)\right]+2 V^{2} X_{0}^{(1)} \frac{\partial R_{0}}{\partial \lambda}+\right. \\
\left.+V^{2} \sum_{k=0}^{N-1} \frac{\partial h_{k}}{\partial \lambda}\left(X_{1}-X_{2}\right)+2 V^{2} \frac{\partial R_{0}}{\partial \lambda} \sum_{k=0}^{N-1} h_{k}\left(X_{1}^{(1)}-X_{2}^{(1)}\right)\right\}
\end{gathered}
$$




$$
\begin{gathered}
X_{0}^{(1)}=\exp \left(-\frac{1}{R_{0}^{2}}\right) \sum_{q=0}^{2 m-2} D_{q} \sum_{p=0}^{l+q} \frac{(l+q) !}{p ! R_{0}^{2 p+3}}\left(1-p R_{0}^{2}\right) ; \\
X_{1}^{(1)}=\exp \left(-\frac{k^{2}}{N^{2} R_{0}^{2}}\right) \sum_{q=0}^{2 m-2} D_{q} \sum_{p=0}^{l+q} \frac{(l+q) !}{p ! R_{0}^{2 p+3}} \frac{k^{2 p}}{N^{2 p}}\left(\frac{k^{2}}{N^{2}}-p R_{0}^{2}\right) ; \\
X_{2}^{(1)}=\exp \left(-\frac{(k+1)^{2}}{N^{2} R_{0}^{2}}\right) \sum_{q=0}^{2 m-2} D_{q} \sum_{p=0}^{l+q} \frac{(l+q) !}{p ! R_{0}^{2 p+3}} \frac{(k+1)^{2 p}}{N^{2 p}}\left(\frac{(k+1)^{2}}{N^{2}}-p R_{0}^{2}\right),
\end{gathered}
$$

и эквивалентного нормированного радиуса пятна моды:

$$
\begin{gathered}
\frac{\partial R_{0}}{\partial \lambda}=\frac{S_{0} \frac{\partial V^{2}}{\partial \lambda}+\sum_{k=0}^{N-1}\left[\left(S_{0}-S_{1}\right)\left(h_{k} \frac{\partial V^{2}}{\partial \lambda}+V^{2} \frac{\partial h_{k}}{\partial \lambda}\right)\right]}{-2 V^{2} \cdot\left[S_{0}^{(1)}+\sum_{k=0}^{N-1} h_{k}\left(S_{1}^{(1)}-S_{2}^{(1)}\right)\right]} ; \\
S_{0}^{(1)}=\exp \left(-\frac{1}{R_{0}^{2}}\right) \sum_{q=0}^{2 m-2} D_{q} \sum_{p=0}^{l+q} \frac{(l+q) !}{p ! R_{0}^{2 p+3}}\left[\left(1-p R_{0}^{2}\right)^{2}-p R_{0}^{4}\right] ; \\
S_{1}^{(1)}=\exp \left(-\frac{k^{2}}{N^{2} R_{0}^{2}}\right)^{2 m-2} \sum_{q=0}^{(1)} D_{q} \sum_{p=0}^{l+q} \frac{(l+q) ! k^{2 p}}{p ! N^{2 p} R_{0}^{2 p+3}}\left[\left(\frac{k^{2}}{N^{2}}-p R_{0}^{2}\right)^{2}-p R_{0}^{4}\right] ; \\
\left.S_{2}^{2}\right] \\
\left(-\frac{(k+1)^{2}}{N^{2} R_{0}^{2}} \sum_{q=0}^{2 m-2} D_{q} \sum_{p=0}^{l+q} \frac{(l+q) !(k+1)^{2 p}}{p ! N^{2 p} R_{0}^{2 p+3}}\left[\left(\frac{(k+1)^{2}}{N^{2}}-p R_{0}^{2}\right)^{2}-p R_{0}^{4}\right] .\right.
\end{gathered}
$$

Затем, соответственно, перейти к производной постоянной распространения $\partial \beta / \partial \lambda$ :

$$
\frac{\partial \beta^{2}}{\partial \lambda}=-\frac{2 k_{0}^{2} n_{\max }^{2}}{\lambda}+k_{0}^{2} \frac{\partial n_{\max }^{2}}{\partial \lambda}-\frac{1}{a^{2}} \frac{\partial U^{2}}{\partial \lambda} ;
$$

и далее получить конечное аналитическое выражение для модовой задержки $t_{d}$, обратно пропорциональной групповой скорости $v_{g}$, направляемой моды $L P_{l m}$ заданного порядка в виде 


$$
t_{d}=\frac{1}{v_{g}}=-\frac{\lambda^{2}}{4 \pi \beta c} \frac{\partial \beta^{2}}{\partial \lambda}=-\frac{\pi}{k_{0}^{2} \beta c} \frac{\partial \beta^{2}}{\partial \lambda} .
$$

Для учета спектральной зависимости показателя преломления применяется известная формула Селлмейера [26]:

$$
n(\lambda)=\sqrt{1+\sum_{i=1}^{3} \frac{A_{i} \lambda^{2}}{\lambda^{2}-B_{i}^{2}}},
$$

где $A_{i}$ и $B_{i}$ - коэффициенты Селлмейера.

В данной работе предлагается воспользоваться представленным в [30] подходом, который базируется на переходе к производным квадрата показателя преломления, в частности $\partial n^{2} / \partial \lambda$. Это существенно упрощает вывод выражений для производных параметров профиля, например $\Delta$ и $h_{k}$, а также нормированной частоты $V$, которые приводятся к следующему виду:

$$
\begin{gathered}
\frac{\partial \Delta}{\partial \lambda}=\frac{1}{2 n_{0}^{2}}\left[(1-2 \Delta) \frac{\partial n_{0}^{2}}{\partial \lambda}-\frac{\partial n_{N}^{2}}{\partial \lambda}\right] ; \\
\frac{\partial h_{k}}{\partial \lambda}=\frac{1}{n_{0}^{2}-n_{N}^{2}}\left[\left(1-h_{k}\right) \frac{\partial n_{0}^{2}}{\partial \lambda}-\frac{\partial n_{k}^{2}}{\partial \lambda}+h_{k} \frac{\partial n_{N}^{2}}{\partial \lambda}\right] ; \\
\frac{\partial V^{2}}{\partial \lambda}=a^{2} k^{2}\left[\frac{\partial n_{0}^{2}}{\partial \lambda}-\frac{\partial n_{N}^{2}}{\partial \lambda}-\frac{2}{\lambda}\left(n_{0}^{2}-n_{N}^{2}\right)\right],
\end{gathered}
$$

где

$$
\frac{\partial n^{2}}{\partial \lambda}=-2 \lambda \sum_{i=1}^{3} \frac{A_{i} B_{i}^{2}}{\left(\lambda^{2}-B_{i}^{2}\right)^{2}} .
$$

В свою очередь поиск коэффициентов Селлмейера для не табулированных значений концентрации примеси осуществляется с помощью метода, представленного в работе [31].

Таким образом, моделирование волокон LDMDF включает в себя следующие этапы. Предварительно выбирается опорное значение модовой задержки $t_{\mathrm{BASE}}$. В рамках данной работы это предлагается сделать по результатам анализа диаграммы DMD, построенной для направляемых мод заданного порядка многомодовых OB, оптимизиро- 
ванных для совместной работы с лазерными источниками, категорий OM2+...ОМ4, на оптической несущей $\lambda=1550$ нм. Здесь расчет параметров модового состава также осуществляется на основе представленного ОМПГ. Число мод $M$, используемых при формировании диаграммы DMD, как было показано выше, определяется условиями ввода сигнала с выхода лазера, составом поперечных мод излучения, которое формируется непосредственно на выходе пигтейла источника, а также прогнозом появления новых модовых составляющих, обусловленного нерегулярной структурой ОВ и наличием микро- и макроизгибов волокон в кабеле, неизбежно возникающих при инсталляции ВОЛП.

Для минимизации целевой функции (4) предлагается использовать симплексный метод Нелдера - Мида, эффективность которого была продемонстрирована в известных работах А.Н. Боголюбова и А.Г. Свешникова, посвященных решению задач синтеза волоконных световодов с заданными характеристиками, например [32] и др.

\section{4. Результаты синтеза профиля показателя преломления волокон LDMDF}

Ниже представлены некоторые результаты предварительной апробации предложенного подхода к моделированию волокон LDMDF для передачи оптических сигналов в маломодовом режиме в телекоммуникационных диапазонах «С» и «L», что соответствует интервалу длин волн $\lambda=1530 \ldots 1625$ нм.

В качестве базового многомодового ОВ для определения границ диапазона выбора опорного значения модовой задержки $t_{\text {BASE }}$ использовалось многомодовое ОВ 50/125 Corning® категории ОМ2+/OM3, профиль показателя преломления которого, восстановленный на основе протоколов измерения промышленного образца анализатором EXFO NR-9200HR [33], представлен на рис. 1. Данный профиль можно рассматривать как наиболее близкий к гладкому степенному. Однако, как видно из рис. $1, a$, в целом он не является $\alpha$-профилем и, кроме того, отличается от последнего наличием слабых локальных флуктуаций показателя преломления, что видно на рис. 1, б, где приведен увеличенный фрагмент, соответствующий центральной области сердцевины этого ОВ.

Предварительный анализ многомодового ОВ OM2+/OM3 с помощью ОМПГ показал, что на оптической несущей $\lambda=1550$ нм данный 

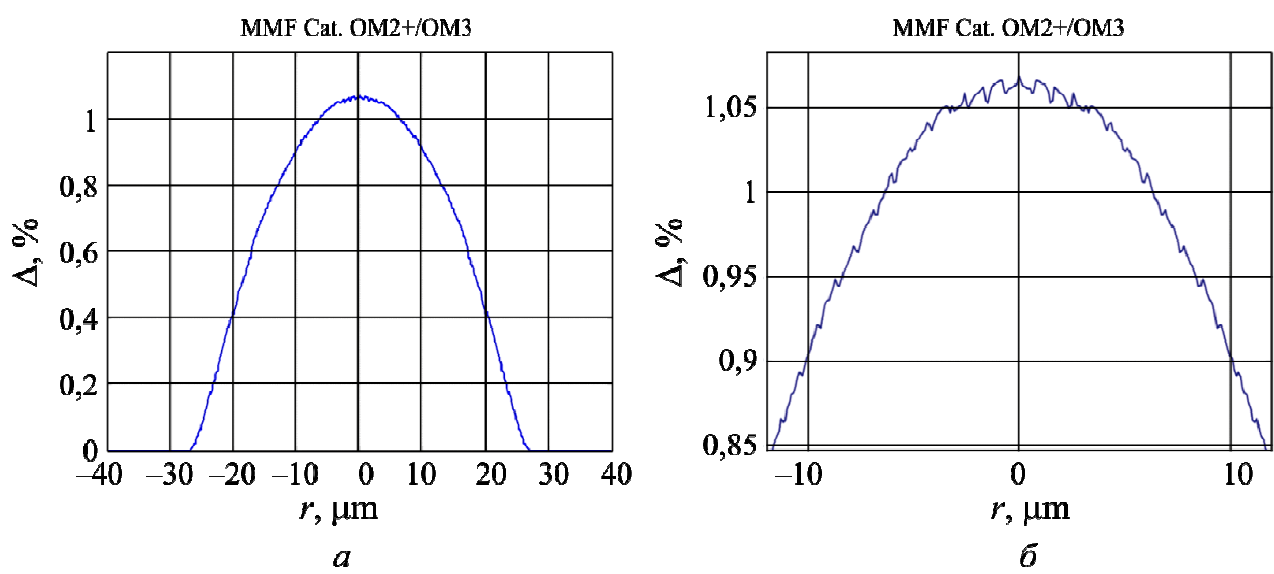

Рис. 1. Градиентный профиль со слабыми флуктуациями показателя преломления промышленного образца многомодового ОВ 50/125 категории ОМ2+/ОМ3: $a$ - общий вид; $\sigma$ - увеличенный фрагмент центральной области сердцевины

волоконный световод в целом поддерживает распространения направляемых мод $L P_{l m}$ вплоть до радиального порядка $m=5$. Далее, согласно проведенному расчету коэффициентов связи мод на стыке одномодового согласующего световода, представляющего собой стандартное одномодовое ОВ Рекомендации МСЭ-T G.652, и рассматриваемого многомодового ОВ в условиях центрированного ввода основной моды $L P_{01}$ с выхода одномодового лазера на этой же длине волны $\lambda=1550$ нм, большая часть мощности данной основной моды передается трем направляемым модам многомодового ОВ $L P_{l m}(l=0 ; m=1 \ldots 3)$, для которых, как было отмечено выше, оптический фактор ограничения составляет не менее $P_{c o}^{(l m)} \geq 0,5$, а нормированная амплитуда не менее $A \geq 0,1$. Наконец, последующее моделирование процессов взаимодействия и смешения модовых составляющих, выполненное на основе модели кусочно-регулярной маломодовой ВОЛП, представленной в работе [17], позволило выявить возбуждение новых направляемых мод $L P_{l m}$ вплоть до 3-го азимутального и также 3-го радиального порядков. Таким образом, построение базовой диаграммы DMD, определяющей границы выбора опорного значения $t_{\mathrm{BASE}}$ и последующий синтез искомого профиля показателя преломления OB LDMDF, осуществляется для 12 направляемых мод $L P_{l m}(l=0 \ldots 3 ; m=1 \ldots 3)$. В качестве опорного значения модовой задержки было выбрано $t_{\mathrm{BASE}}=4,9257$ мкс/км из диаграммы DMD, построенной для многомодового OB категории $\mathrm{OM} 2+/ \mathrm{OM} 3$. 


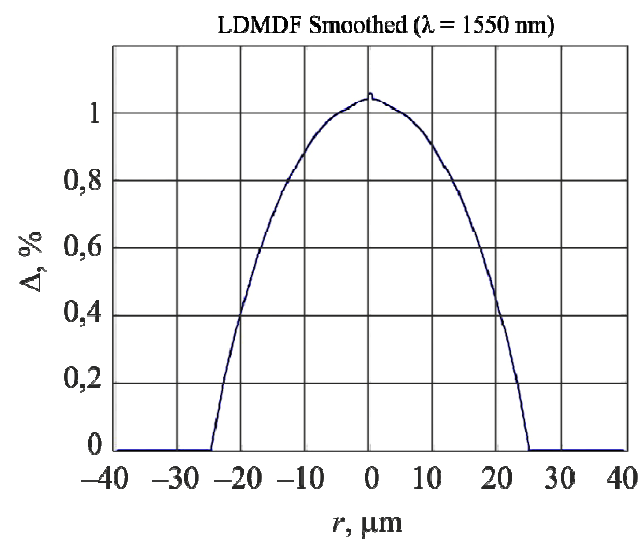

Рис. 2. Синтезированный профиль показателя преломления многомодового OB 50/125 LDMDF, оптимизированный для передачи оптических сигналов на длине волны $\lambda=1550$ нм

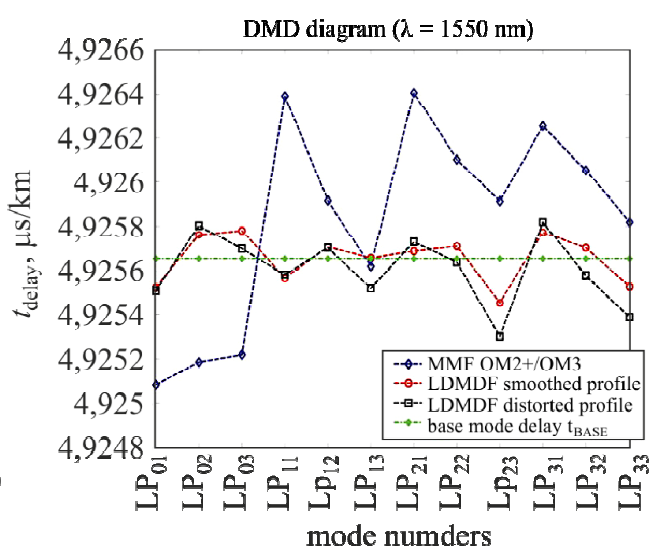

Рис. 3. Диаграммы DMD для исходного профиля ОВ ОМ2+/OM3, синтезированного профиля LMDF, профиля LMDF с внесенными искажениями, а также опорная модовая задержка $t_{\mathrm{BASE}}$

Полученный в результате минимизации целевой функции (4) профиль показателя преломления многомодового OB 50/125 LDMDF, ориентированный на передачу маломодовых оптических сигналов на длине волны $\lambda=1550$, представлен на рис. 2 . Для сравнения на рис. 3 представлены диаграмма DMD для профиля многомодового OB OM2+/OM3, опорное значение модовой задержки $t_{\mathrm{BASE}}$ и диаграмма DMD для синтезированного профиля показателя преломления OB LDMDF, построенные для длины волны $\lambda=1550$ нм. Максимальное отклонение диаграммы относительно заданного опорного значения не превышает $0,0045 \%$.

Современные технологии производства заготовок градиентных $\mathrm{OB}$, в частности MCVD и тем более PCVD, позволяют формировать профили показателя преломления из более 1000 слоев. Таким образом, можно предположить, что при изготовлении OB LDMDF общая форма полученного профиля будет воспроизведена с достаточно высокой точностью. Однако, как было показано выше, даже для градиентных профилей волокон последнего поколения характерны локальные флуктуации показателя преломления (см. рис. 1, б). Последние, как показал анализ протоколов измерения профилей промышленных образцов многомодовых ОВ OM2+/OM3, достаточно хорошо описываются нормальным законом распределения случайной величины. 
Для моделирования данного эффекта к синтезированной форме профиля LDMDF были добавлены локальные отклонения показателя преломления, согласно, как было отмечено выше, нормальному закону распределения. Здесь в качестве локального математического ожидания на радиальной координате $r_{k}$ использовалось соответствующее значение показателя преломления $n_{k}$ синтезированного гладкого профиля LDMDF, в то время как среднеквадратическое отклонение выбиралось намеренно завышенным относительно полученных в результате анализа профилей промышленных образцов волокон OM2+/OM3 значений и составило $5 \cdot 10^{-5}$. Общий вид профиля показателя преломления OB LDMDF с внесенными локальными искажениями и его увеличенный фрагмент, соответствующий центральной области сердцевины, представлены на рис. $4, a, \sigma$.

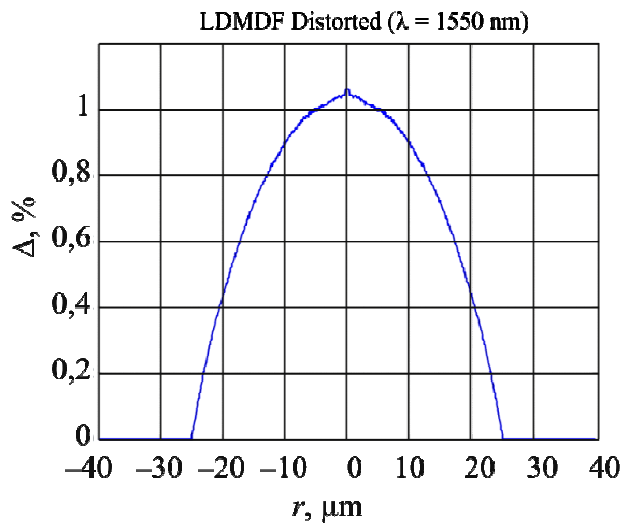

$a$

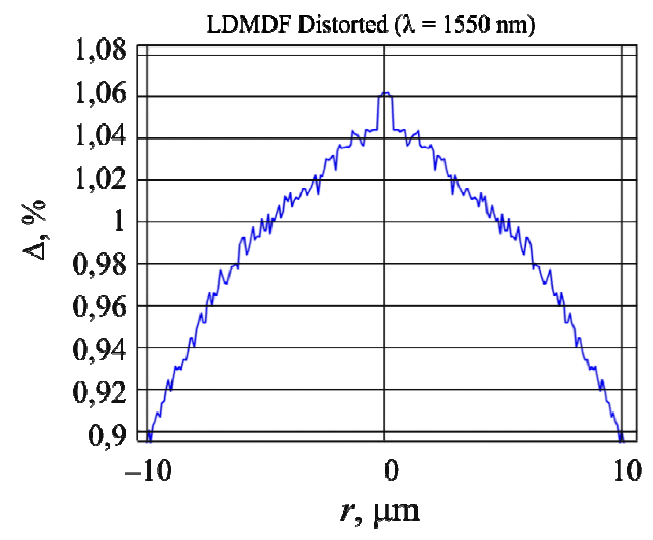

$\sigma$

Рис. 4. Синтезированный профиль показателя преломления многомодового ОВ 50/125 LDMDF с внесенными локальными флуктуациями показателя преломления:

$a$ - общий вид; $\sigma$ - увеличенный фрагмент центральной области сердцевины

Для указанного профиля LDMDF с внесенными искажениями была также построена диаграмма DMD на оптической несущей $\lambda=1550$ и приведено сопоставление с опорным значением модовой задержки $t_{\mathrm{BASE}}$ (см. также рис. 3). В этом случае максимальное отклонение диаграммы по-прежнему принимает достаточно малые значения, но уже не превышает, в отличие от гладкого модельного профиля, 0,0075\%.

С точки зрения оценки потенциальных возможностей использования приложения многомодовых OB LDMDF на транспортных сетях связи нового поколения был проведен расчет DMD в телекоммуника- 
ционных волновых диапазонах «С»и «L» $\lambda=1530 \ldots 1625$ нм). В данном случае оценивалась максимальная модовая задержка относительно среднего времени поступления модового состава $L P_{l m}(l=0 \ldots 3$; $m=1 \ldots 3)$ на приемную сторону ВОЛП. Рассматривались сглаженный профиль показателя преломления OB LDMDF и профиль с внесенными локальными искажениями. Также для сравнения аналогичные расчеты были проведены и для спектральной характеристики DMD многомодовых ОВ ОМ2+/ОМ3. Результаты расчетов представлены на рис. 5, $а$. Анализ полученных данных показывает следующее. Локальные флуктуации показателя преломления профиля OB LDMDF могут существенно увеличить значение DMD - от 35 до $65 \%$ - в исследуемом спектральном диапазоне. В то же время полученный в результате синтеза профиль ОВ LDMDF даже с локальными отклонениями обеспечивает общее снижение DMD модового состава $L P_{l m}(l=0 \ldots 3 ; m=1 \ldots 3)$ в $1,8-3,0$ раза по сравнению с DMD OB категории OM2+/OM3, при этом сглаженный профиль LDMDF без флуктуаций уменьшает значение этого параметра в 2,5-5 раз относительно ОВ ОМ2+/OM3.
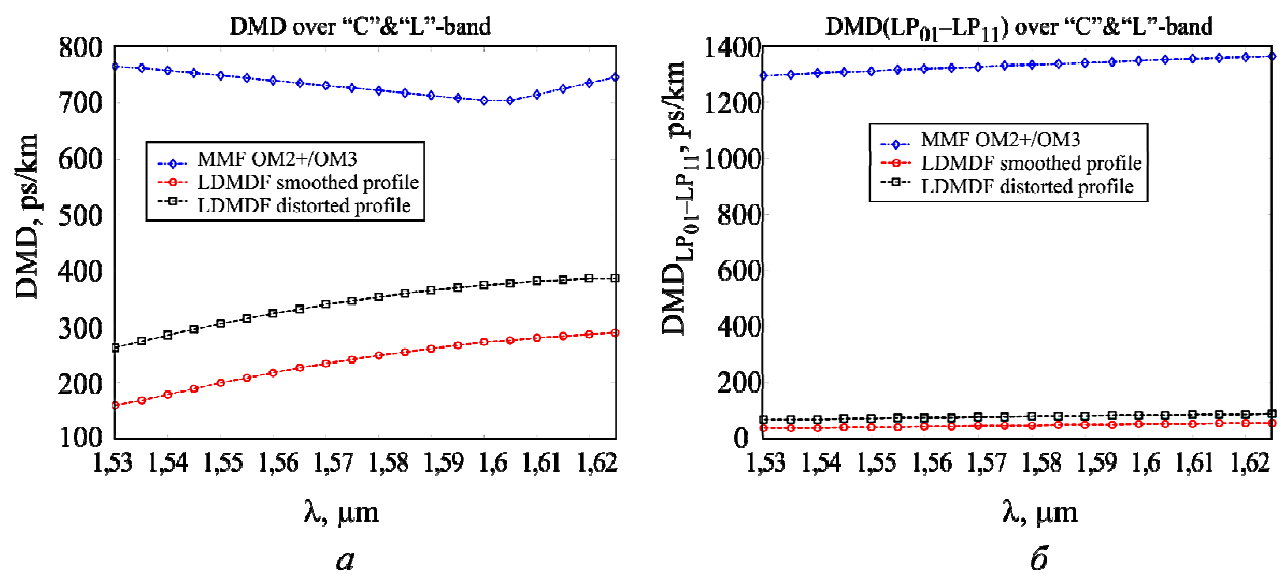

Рис. 5. Спектральные характеристики DMD в диапазонах «C» и «L»: $a$ - максимальная модовая задержка относительно среднего времени поступления модового состава $L P_{l m}(l=0 . . .3 ; m=1 . .3)$ на приемную сторону; $\sigma$ - задержка между модами $L P_{01}$ и $L P_{11}$

Следует также отметить, что в ряде случаев (в частности, при строго центрированном вводе с выравниванием диаметра пятна основной моды) в процессе распространения оптического импульса, исходный модовый состав которого ограничен одной основной модой $L P_{01}$, 
наиболее активно взаимодействуют упомянутая основная мода $L P_{01}$ и мода $L P_{11}$, которые фактически и переносят основную часть мощности сигнала. Поэтому отдельный интерес представляет оценка DMD между указанными модовыми компонентами. Спектральные характеристики DMD $L P_{01}-L P_{11}$, построенные как для многомодового ОВ OM2+/OM3, так и волокон LDMDF с синтезированным сглаженным профилем и профилем с внесенными локальными искажениями, представлены на рис. 5, б. В этом случае, согласно полученным результатам расчета, переход на OB LDMDF даже с искаженным профилем показателя преломления обеспечивает снижение задержки между указанными модами более чем на порядок.

\section{5. Заключение}

В работе представлен альтернативный поход к моделированию профиля показателя преломления кварцевых OB LDMDF с увеличенным до 50 мкм диаметром сердцевины и при этом оптимизированным для передачи сигналов в телекоммуникационных «C» и «L» волновых диапазонах. Представлены предварительные результаты апробации предложенного метода. Полученный в результате синтеза профиль показателя преломления OB LDMDF демонстрирует достаточно высокие потенциальные возможности предложенного подхода с точки зрения минимизации DMD при использовании OB типовой конструкции с увеличенным до 50 мкм диаметром сердцевины и специализированным профилем показателя преломления на высокоскоростных транспортных сетях связи нового поколения. Так, значение DMD для таких OB LDMDF в спектральных диапазонах «C» и «L» удается уменьшить, по сравнению с типовыми ОВ категории ОМ2+/OM3, в 1,8-3,0 раза даже при наличии достаточно сильных локальных флуктуаций показателя преломления. Также спектральная характеристика DMD для OB LDMDF носит более монотонный характер по сравнению с ОВ ОМ2+/ОМ3. Это позволяет сделать предположение о возможности компенсации дисперсионных искажений при распространении сигналов в таких волокнах с точки зрения диаграммы DMD не только на отдельной оптической несущей, но и в заданном спектральном диапазоне. Вместе с тем очевидно, что требуется проведение дополнительных исследований, в том числе по выбору опорного значения модовой задержки $t_{\text {BASE }}$ с точки зрения снижения DMD на отдельной длине волны, а также пе- 
реходу к синтезу профиля показателя преломления OB LDMDF не только с плоской диаграммой DMD на модах заданного порядка для отдельной оптической несущей, но и плоской спектральной характеристикой DMD в заданном диапазоне длин волн.

\section{Список литературы}

1. Mitra P.P., Stark J.B. Nonlinear limits to the information capacity of optical fibre communications // Nature. - 2001. - Vol. 411. - P. 1027-1030.

2. Innovative future optical transport network technologies / T. Morioka [et al.] // NTT Technical Review. - 2011. - Vol. 9, no. 8. - URL: https: //www.ntt-review.jp/archive/ntttechnical.php? contents = ntr201108fa6.html.

3. Capacity Limits of Optical Fiber Networks / R.-J. Essiambre [et al.] // Journal of Lightwave Technology. - 2010. - Vol. 28, no. 4. - P. 662-701.

4. Essiambre R.-J., Tkach R.W. Capacity trends and limits of optical communication networks // Proceedings of IEEE. - 2012. - Vol. 100, no. 5. P. 1035-1055.

5. The nonlinear Shannon limit and the need for new fibres / D. Ellis [et al.] // Proceedings of SPIE. - 2012. - Vol. 8434. - P. 84340H-1-84340H-11.

6. Hirano M. Future of transmission fiber // IEEE Photonics Journal. 2011. - Vol. 3, no. 2. - P. 316-319.

7. Richardson D.J., Fini J.M., Nelson L.E. Space-division multiplexing in optical fibers // Nature Photonics. - 2013. - Vol. 7, no. 5. - P. 354-362.

8. Morioka T. Recent progress in space-division multiplexed transmission technologies // OFC/NFOEC Technical Digest. - 2013. - OW4F.2. P. OW4F.2-1-OW4F.2-4.

9. Software defined networking (SDN) over space division multiplexing (SDM) optical networks: features, benefits and experimental demonstration / N. Amaya [et al.] // Optics Express. - 2014. - Vol. 22, no. 3. P. 3638-3647.

10. Ultra-high-density spatial division multiplexing with a fewmode multicore fibre / R.G.H. van Uden [et al.] // Nature Photonics. 2014. - No. 10. - P. 1-6.

11. Yamamoto Y., Hirano M., Sasaki T. Low-loss and low-nonlinearity pure-silica-core fiber for large capacity transmission // SEI Technical Review. - 2013. - Vol. 76. - P. 63-68. 
12. Mode division multiplexing over $96 \mathrm{~km}$ of few mode fiber using coherent 6×6 MIMO processing / R. Ryf [et al.] // Journal of Lightwave Technology. - 2012. - Vol. 30, no. 4. - P. 521-531.

13. Space-division multiplexed high-speed superchannel transmission over few-mode fiber / A. Li [et al.] // Journal of Lightwave Technology. 2012. - Vol. 30, no. 24. - P. 3953-3964.

14. $73.7 \mathrm{~Tb} / \mathrm{s}(96 \times 3 \times 256-\mathrm{Gb} / \mathrm{s})$ mode-division-multiplexed DP-16QAM transmission with inline MM-EDFA / V.A.J.M. Sleiffer [et al.] // Optics Express. - 2012. - Vol. 20, no. 26. - P. B428-B438.

15. Теумин И.И. Волноводы оптической связи. - М.: Связь, 1978. - $178 \mathrm{c}$.

16. Андрушко Л.М., Вознесенский В.А., Каток В.Б. Справочник по волоконно-оптическим линиям связи. - Киев: Тэхника, 1988. - 239 с.

17. Bourdine A. Modeling and simulation of piecewise regular multimode fiber links operating in a few-mode regime. Advances in Optical Technologies. - 2013. - Vol. 2013. - URL: http: //www.hindawi.com/journals/aot/2013/469389.

18. Бурдин А.В. Маломодовый режим передачи оптических сигналов по многомодовым волокнам: приложения в современных инфокоммуникациях. - Самара: ПГУТИ, 2011. - 274 с.

19. Patent 4286979 United States Patent, IPC Classification C03B37/07, C03B37/075, G01N21/00. Fabrication of optical fibers using differential mode-group delay measurement / M.J. Buckler, R. Kummer, S.C. Mettler, M. Miller, Bell Telephone Laboratories Inc., USA, - No. 19800162263, priority date 23.06.1980; publication date 01.09.1981.

20. Stone F.T., Ritger A.J., Head E.D. The use of a quantitative differential mode delay technique to improve fiber bandwidth // IEEE Journal of Lightwave Technology. - 1983. - Vol. LT-1, no. 4. - P. 585-587.

21. Patent US 2002/0197038 United States Patent, IPC Classification G02B6/18, G02B6/16. Laser optimized multimode fiber and method for use with laser and system employing same / J.S. Abbot, D.E. Harsbarger, Corning Inc., USA, - No. 10/217812, priority date 12.08.2002; publication date 26.12.2002.

22. Patent WO 2007/043060 A1 World Intellectual Property Organization, IPC Classification C03B37/025, C03B37/07. Optical fiber having higher bandwidth and method for producing the same / P. Bangalore Krishnaswamy, S. Dutta, S.R. Panneerselvam, S.K. Nageswaran, Sterlite Optical Technolo- 
gies Ltd., India, - No PCT/IN2005/000354, priority date 07.10.2005; publication date 19.04.2007.

23. Patent US 6574403 B1 United States Patent, IPC Classification G02B6/02, G02B6/16. Apparatus and method for improving bandwidth of multimode fibers / S.E. Golowich, S.L. Jones, A.J. Ritger, K.S. Thornburg, Fitel USA Corp., USA, - No 09/575867, priority date 17.05.2002; publication date 03.06.2003.

24. New generation of broad wavelength window multimode fibres / F.J. Achten [et al.] // Proceedings of 30-th European Conference on Optical Communication (ECOC) Stockholm, Sweden, 2004. - Stockholm, 2004 Vol. 4. - P. Th 3.3.3-1-Th 3.3.3-3.

25. High-speed transmission in multimode fibers / R.E. Freund [et al.] // IEEE Journal of Lightwave Technology. - 2010. - Vol. 28, no. 4. P. 569-586.

26. Адамс М. Введение в теорию оптических волноводов: пер. с англ. - М.: Мир, 1984. - 512 с.

27. Bourdine A.V. Method for chromatic dispersion estimation of high-order guided modes in graded index single-cladding fibers // Proceedings of SPIE. - 2007. - Vol. 6605. - P. 660509-1-660509-13.

28. Снайдер А., Лав Дж. Теория оптических волноводов: пер. с англ. - М.: Радио и связь, 1987. - 656 с.

29. Градштейн И.С., Рыжик И.М. Таблицы интегралов. - М.: Физматгиз, 1962. - 1100 с.

30. Burdin V., Andreev R., Praporshchikov D. Model and algorithm of optimization of a refractive index profile of single-mode fibers for optical communication networks // Proceedings of SPIE. - 2004. - Vol. 5485 - - P. 63-74.

31. Бурдин В.А. Методы определения коэффициентов формулы Селлмейера в задачах анализа дисперсионных характеристик кварцевых оптических волокон // Инфокоммуникационные технологии.2006. - № 2. - С. 30-34.

32. Боголюбов А.Н., Буткарев И.А., Свешников А.Г. Синтез волоконных световодов // Радиотехника. - 2004. - № 12. - С. 4-12.

33. Бурдин А.В., Яблочкин К.А. Исследование дефектов профиля показателя преломления многомодовых оптических волокон кабелей связи // Инфокоммуникационные технологии. - 2010. - № 2. - С. 22-27. 


\section{References}

1. Mitra P.P., Stark J.B. Nonlinear limits to the information capacity of optical fibre communications. Nature, 2001, vol. 411, pp. 1027-1030.

2. Morioka T. [et al.]. Innovative future optical transport network technologies. NTT Technical Review, 2011, vol. 9, no. 8, available at: https:// www.ntt-review.jp/archive/ntttechnical.php?contents=ntr201108fa6.html.

3. Essiambre R.-J. [et al.]. Capacity Limits of Optical Fiber Networks. Journal of Lightwave Technology, 2010, vol. 28, no. 4, pp. 662-701. DOI: 10.1109/JLT.2009.2039464.

4. Essiambre R.-J., Tkach R.W. Capacity trends and limits of optical communication networks. Proceedings of IEEE, 2012, vol. 100, no 5, pp. 1035-1055. DOI: 10.1109/JPROC.2012.2182970

5. Ellis D. [et al.]. The nonlinear Shannon limit and the need for new fibres. Proceedings of SPIE, 2012, vol. 8434, pp. 84340H-1-84340H-11. DOI: $10.1117 / 12.928093$.

6. Hirano M. Future of transmission fiber. IEEE Photonics Journal, 2011, vol. 3, no 2, pp. 316-319. DOI: 10.1109/JPHOT.2011.2130519.

7. Richardson D.J., Fini J.M., Nelson L.E. Space-division multiplexing in optical fibers. Nature Photonics, 2013, vol. 7, no. 5, pp. 354-362. DOI: 10.1038/nphoton.2013.94. 8.

8. Morioka T. Recent progress in space-division multiplexed transmission technologies. OFC/NFOEC Technical Digest, 2013, OW4F.2, pp. OW4F.2-1- OW4F.2-4.

9. Amaya N. [et al.] Software defined networking (SDN) over space division multiplexing (SDM) optical networks: features, benefits and experimental demonstration. Optics Express, 2014, vol. 22, no 3, pp. 3638-3647.

10. Uden R.G.H. van [et al.]. Ultra-high-density spatial division multiplexing with a few-mode multicore fibre. Nature Photonics, 2014, no. 10, pp. 1-6. DOI: 10.1038/NPHOTON.2014.243.

11. Yamamoto Y., Hirano M., Sasaki T. Low-Loss and Low-Nonlinearity Pure-Silica-Core Fiber for Large Capacity Transmission. SEI Technical Review, 2013, vol. 76, pp. 63-68.

12. Ryf R. [et al.]. Mode division multiplexing over $96 \mathrm{~km}$ of few mode fiber using coherent $6 \times 6 \mathrm{MIMO}$ processing. Journal of Lightwave Technology, 2012, vol. 30, no 4, pp. 521-531. DOI: 10.1109/JLT.2011.2174336.

13. Li A. [et al.]. Space-division multiplexed high-speed superchannel transmission over few-mode fiber. Journal of Lightwave Technology, 2012, vol. 30, no. 24, pp. 3953-3964. DOI: 10.1109/JLT.2012.2206797. 
14. Sleiffer V.A.J.M. [et al.]. $73.7 \mathrm{~Tb} / \mathrm{s}(96 \times 3 \times 256 \mathrm{~Gb} / \mathrm{s})$ mode-divisionmultiplexed DP-16QAM transmission with inline MM-EDFA. Optics Express, 2012, vol. 20, no. 26, pp. B428- B438.

15. Teumin I.I. Volnovody opticheskoi sviazi [Optical waveguides for optical communications]. Moscow: Sviaz', 1978, 171 p.

16. Andrushko L.M., Voznesenskii V.A., Katok V.B. Spravochnik po volokonno-opticheskim liniiam sviazi [Guide on fiber optic communication links]. Kiev: Tekhnika, 1988. 239 p.

17. Bourdine A. Modeling and simulation of piecewise regular multimode fiber links operating in a few-mode regime. Advances in Optical Technologies, 2013, vol. 2013, available at: http://www.hindawi.com/journals/aot/2013/469389.

18. Bourdine A.V. Malomodovyi rezhim peredachi opticheskikh signalov po mnogomodovym voloknam: prilozheniia $v$ sovremennykh infokommunikaciiah [Few mode regime of optical signal transmission over multimode fibers: applications in modern infocommunications]. Samara, 2011, 274 p.

19. Buckler M.J., Kummer R., Mettler S.C., Miller M. Fabrication of optical fibers using differential mode-group delay measurement. Patent 4286979 United States Patent. IPC Classification C03B37/07, C03B37/075, G01N21/00. Bell Telephone Laboratories Inc., USA, No 19800162263, priority date 23.06.1980; publication date 01.09.1981.

20. Stone F.T., Ritger A.J., Head E.D. The use of a quantitative differential mode delay technique to improve fiber bandwidth. IEEE Journal of Lightwave Technology, 1983, vol. LT-1, no. 4, pp. 585-587. DOI: 10.1109/JLT.1983.1072155.

21. Abbot J.S., Harsbarger D.E. Laser optimized multimode fiber and method for use with laser and system employing same. Patent US 2002/0197038 United States Patent. IPC Classification G02B6/18, G02B6/16. Corning Inc., USA, No 10/217812, priority date 12.08.2002; publication date 26.12.2002.

22. Bangalore Krishnaswamy P., Dutta S., Panneerselvam S.R., Nageswaran S.K. Optical fiber having higher bandwidth and method for producing the same. Patent WO 2007/043060. A1 World Intellectual Property Organization, IPC Classification C03B37/025, C03B37/07. Sterlite Optical Technologies Ltd., India. No PCT/IN2005/000354, priority date 07.10.2005; publication date 19.04.2007.

23. Golowich S.E., Jones S.L., Ritger A.J., Thornburg K.S. Apparatus and method for improving bandwidth of multimode fibers Patent $U S$ 6574403 B1 United States Patent. IPC Classification G02B6/02, G02B6/16. Fitel USA Corp., USA. No 09/575867, priority date 17.05.200; publication date 03.06.2003. 
24. Achten F.J. [et al.]. New generation of broad wavelength window multimode fibres. Proceedings of 30th European Conference on Optical Communication (ECOC) Stockholm, Sweden, 2004, vol. 4, pp. Th 3.3.3-1-Th 3.3.3-3.

25. Freund R.E. [et al.]. High-speed transmission in multimode fibers. IEEE Journal of Lightwave Technology, 2010, vol. 28, no 4, pp. 569-586. DOI: 10.1109/JLT.2009.2030897.

26. Adams M. Vvedenie v teoriiu opticheskikh volnovodov [An introduction to optical waveguides]. Moscow: Mir, 1984, 512 p.

27. Bourdine A.V. Method for chromatic dispersion estimation of high-order guided modes in graded index single-cladding fibers. Proceedings of SPIE, 2007, vol. 6605, pp. 660509-1-660509-13. DOI: 10.1117/12.728467.

28. Snaider A., Lav Dzh. Teoriia opticheskikh volnovodov [Optical waveguide theory]. Moscow: Radio I zviaz', 1987, 656 p.

29. Gradshtein I.S., Ryzhik I.M. Tablitsi integralov [Tables of integrals]. Moscow, Fizmatgiz, 1962. 1100 p.

30. Burdin V., Andreev R., Praporshchikov D. Model and algorithm of optimization of a refractive index profile of single-mode fibers for optical communication networks. Proceedings of SPIE, 2004, vol. 5485, pp. 63-74. DOI: $10.1117 / 12.564927$.

31. Burdin V.A. Metody opredeleniia koeffitsientov formuly Sellmeiera $v$ zadachah analiza dispersionnykh kharakteristik kvartsevykh opticheskikh volokon [Methods for computation of Sellmeier coefficients for dispersion analysis of silica optical fibers]. Infokommunikatsionnye tekhnologii, 2006, no. 2, pp. 30-34.

32. Bogoliubov A.N., Butkarev I.A., Sveshnikov A.G. Sintez volokonnykh svetovodov [Synthesis of optical fibers]. Radiotekhnika, 2004, no. 12 , pp. 4-12.

33. Burdin A.V., Iablochkin K.A. Issledovanie defektov profilia pokazatelia prelomleniia mnogomodovykh opticheskikh volokon kabelei sviazi [Research of refractive index profile defects multimode optical fibers of communication cables]. Infokommunikatsionnye tekhnologii, 2010, no. 2, pp. 22-27.

Получено 26.11.2014 